\title{
Primer registro en Chile de Antarctophthirus microchir (Anoplura) en lobo marino común (Otaria flavescens) ${ }^{\#}$
}

\author{
First record of Antarctophthirus microchir (Anoplura) in the southern sea lion \\ (Otaria flavescens) in Chile \\ A Crovetto ${ }^{a^{*}}, \mathbf{R}$ Franjola ${ }^{b}$, R Silva $^{\mathrm{c}}$ \\ anstituto de Zoología, Facultad de Ciencias, Universidad Austral de Chile, Valdivia, Chile. \\ bInstituto de Parasitología, Facultad de Medicina, Universidad Austral de Chile, Valdivia, Chile. \\ 'Instituto de Anatomía, Histología y Patología, Facultad de Medicina, Universidad Austral de Chile, Valdivia, Chile.
}

\section{SUMMARY}

\begin{abstract}
The ectoparasitic fauna associated to marine mammals is scarcely known in Chile. This report deals with a sample of Anoplura parasites collected from adult and juvenile Otaria flavescens, the southern sea lion, at the Los Lagos Region, Chile. The Anoplura were taxonomically determined using the existent keys and they were subsequently described. This is the first record of Antarctophthirus microchir in Chile which, together with what is already known about the geographical distribution and the world evolutionary history of the Otariidae, could represent another evidence of the monophyly of the northern and southern hemisphere Otariidae groups.
\end{abstract}

Palabras clave: lobo marino común, parásito, Anoplura, Antarctophthirus.

Key words: southern sea lion, parasites, Anoplura, Antarctophthirus.

\section{INTRODUCCION}

Muchos insectos están íntimamente asociados con aves y mamíferos dependiendo de ellos para su sobrevivencia. En los animales marinos la asociación puede ser temporal o permanente según las condiciones del terreno donde se ubican los animales, de los tiempos de inmersión, los tiempos que están en tierra y la densidad poblacional (Murray 1976).

Los Mallophaga y Anoplura son ectoparásitos que se encuentran en aves y mamíferos. Los malófagos están ausentes en mamíferos marinos, pero los anopluros están presentes a través de cinco géneros como son: Latagophthirus (nutrias), Echinophthirus (focas), Proechinophthirus (lobos finos), Lepidopththirus (elefante marino y foca monje) y Antarctophthirus (otros otarios y focidos).

Murray (1976) indica que Antarctophthirus microchir es la especie de Anoplura más frecuente en Otariidae, incluida la especie Otaria byronia (Kim y col 1975). O. byronia sería el nombre científico válido para lobo marino común del sur (Oliva 1988), no obstante, Aguayo y col (1998), Fasio y col (2000), Koen Alonso y col (2000), Sepúlveda y col (2001), Szteren y Paez (2002) y Oliva y col (2003) usan el nombre Otaria flavescens para referirse al mismo grupo.

Aceptado: 09.04.2008.

\# Financiamiento DID - UACH Proyecto S-93-23.

* Casilla 567, Valdivia, Chile; ecrovett@uach.cl

\section{MATERIAL Y METODOS}

El estudio se realizó en la isla de Metalqui ubicada en el Parque Nacional Chiloé ( $42^{\circ} 11^{\prime} 30^{\prime \prime}$ S- $74^{\circ} 09^{\prime} \mathrm{W}$ ) donde existen loberías reproductivas de lobos comunes.

La recolección de parásitos se realiza luego de inmovilizar con red y cuerdas a algunos animales adultos que estaban descansando en la playa o regresaban del mar. Se evita cercanía con aves para evitar contaminación.

También se inmovilizaron crías que salían recién del agua o que se encontraban en lugares alejados, tomándolas en brazos para evitar cualquier daño.

Se buscó ectoparásitos en 10 animales adultos y 15 crías en diversas áreas del cuerpo, retirándolos con ayuda de pinzas y guardándolos en alcohol de $70 \%$.

Los ectoparásitos fueron determinados taxonómicamente según la clave indicada por Murray (1976) y observados en microscopio electrónico de barrido, siguiendo la siguiente metodología:

- Sonicación en agua destilada por veinte segundos mediante un equipo de ultrasonido Bandelin Modelo TK.

- Fijación en glutaldehído al $4 \%$.

- Lavado en buffer fosfato pH 7,4-0,1 molar por dos veces por cinco minutos.

- Deshidratación secuencial en batería de alcoholes de $15 \%, 30 \%, 50 \%, 70 \%, 96 \%$ y absoluto (dos veces por cinco minutos en cada alcohol) y luego sumergir en 
mezcla de $50 \%$ de alcohol absoluto y $50 \%$ de acetona pura por un minuto. Finalmente se introduce en acetona pura por un minuto.

- Se hace secado de punto crítico con $\mathrm{CO}_{2}$ en un secador Hitachi modelo HCP-2.

- Se procede a fijar en portamuestras de aluminio.

- Se metaliza en oro paladio con un metalizador Eiko engineering modelo IB-2.

- Se observa en microscopio de barrido Leo Electrón Modelo 420.

\section{RESULTADOS Y DISCUSION}

Se obtuvieron 35 individuos presentes en los 10 adultos y en 8 de las 15 crías, encontrándose preferentemente en la cabeza alrededor de la base del hocico, identificándolos como Antarctophthirus microchir (Troussant y Neumann), de acuerdo a Kim y col (1975) y a la clave de Murray
(1976). Esta determinación se basa en que las antenas poseen cinco segmentos, su cuerpo es moderadamente delgado, su cabeza es más larga que ancha y el tórax es delgado conectado con el abdomen. El macho posee un pseudopene orientado en $\mathrm{V}$ y la hembra posee cerdas de tipo escamoso y cilíndrico (figura $1 \mathrm{~A}, \mathrm{~B}, \mathrm{C}$ y D).

Determinada la especie, se hace una breve descripción del cuerpo del parásito resaltando las cerdas de tipo cilíndrico y escamoso y algunas estructuras corporales:

- Cabeza alargada, con dos cerdas en la zona ventral, mientras que en la dorsal presentan cuatro cerdas, en cada lado se observa una cerda, en la zona frontal hay nueve cerdas, rodeando el aparato bucal. Las antenas presentan cinco artejos, siendo el basal más grande, en el extremo del quinto artejo se observan estructuras que corresponderían a órganos sensoriales (figura 2 E y F).
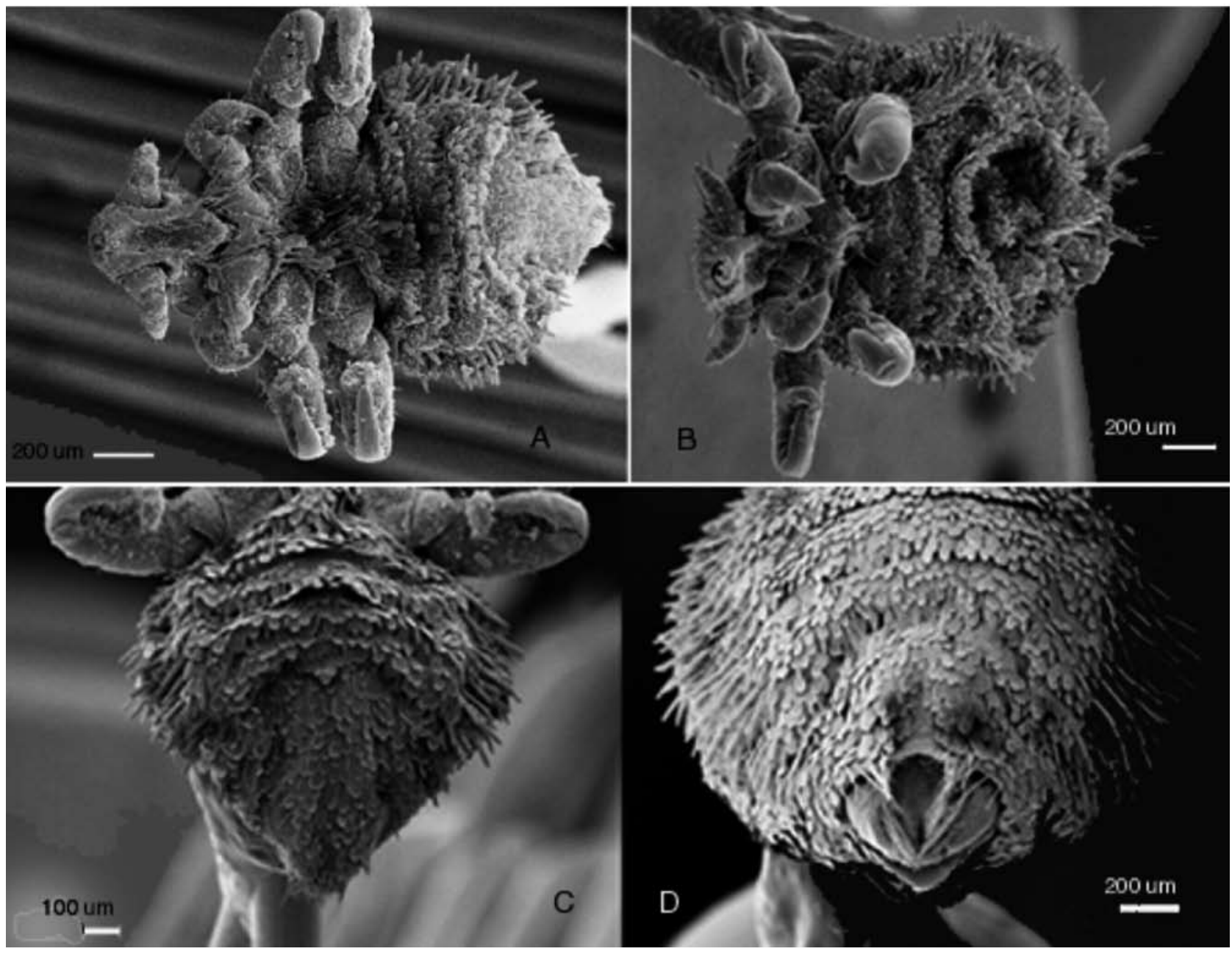

Figura 1. Microscopía de barrido de macho y hembra de Antarctophthirus microchir. A. Vista ventral del macho. B. Vista ventral de la hembra. C. Extremo posterior del macho. D. Extremo posterior de la hembra.

Scanning electron micrographs of male and female of Antarctophthirus microchir. A. Ventral view of male. B. Ventral view of female. C. Posterior end of male. D. Posterior end of female. 
- El tórax, en el dorso, se ensancha hacia la parte posterior, presentando cerdas redondas y escamosas tanto en el dorso como en el vientre. El protórax está menos desarrollado que los mesotórax y metatórax. Existe un surco bien marcado entre el mesotórax y el metatórax. En cada lado del mesotórax y metatórax hay agrupaciones formadas por seis u ocho setas largas. También se observa lateralmente un espiráculo en cada segmento torácico (mesotórax y metatórax) (figura $2 \mathrm{G}$ ). El detalle del espiráculo se muestra en figura $2 \mathrm{H}$.
- La distancia entre los tres pares de patas es similar. El primer par de patas es poco desarrollado, mientras que el segundo y tercer par de patas son fuertes y robustas (figura 1 A y B).

- El abdomen comienza estrecho en parte anterior, ampliándose en el segundo tercio para luego terminar aguzado, tanto en el macho como en la hembra (figura $1 \mathrm{~A}, \mathrm{~B}, \mathrm{C}$ y D). Todo el abdomen está cubierto por setas de tipo escamoso y cilíndrico. En la hembra el poro genital está rodeado por hileras de cerdas muy largas (figura 2 I). El macho en su extremo posterior

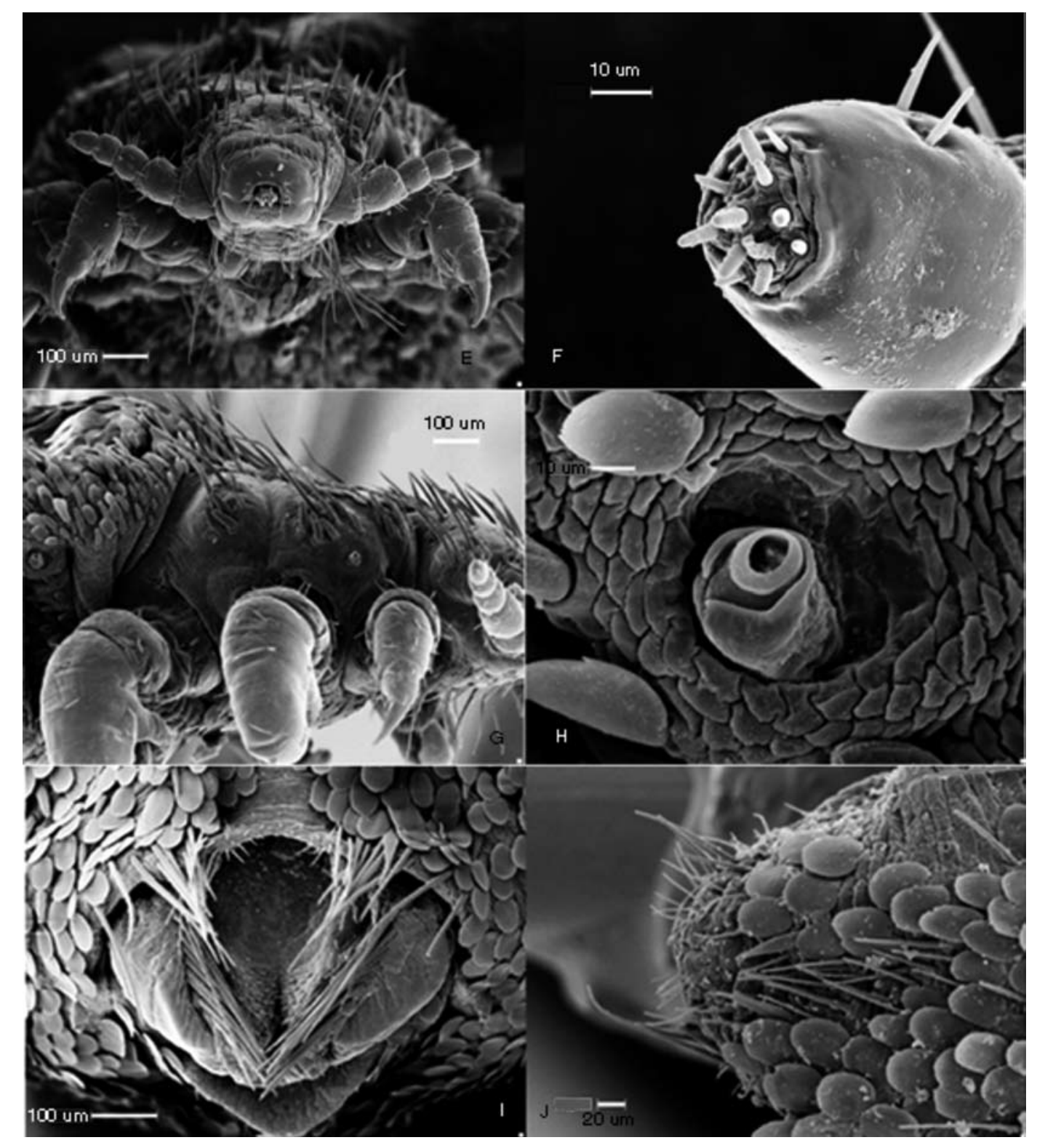

Figura 2. Microscopía de barrido de macho y hembra de Antarctophthirus microchir. E. Vista frontal de la cabeza. F. Vista del extremo de la antena. G. Vista lateral del tórax. H. Espiráculo abdominal. I. Extremo posterior de la hembra. J. Extremo posterior del macho.

Scanning electron micrograph of male and female of Antarctophthirus microchir. E. Frontal view of the head. F. View from the apex antenna. G. Side view of the thorax. H. Opening of tracheal system. I. Female abdomen ending. J. Male abdomen ending. 
también presenta setas largas de ambos tipos, pero en menor número (figura $2 \mathrm{~J}$ ).

- Las cerdas de tipo escamas son de forma ovoide.

Murray y col (1965) y Clay y Moreby (1970) indican para las islas subantárticas presencia de A. microchir para Phocartos hookeri, lo que es corroborado por Gales (1995). Los otros registros de Antarctophthirus se registran para focas con las especies A. ogmorhini, A. lobodontis y A. mawsoni (Clay y Moreby, 1970). Mehlhorn y col (2002) corroboran la primera de ellas para la foca Leptonychotes weddellii. Dailey y col (2005) mencionan para las Islas Galápagos la presencia de A. microchir para Zalophus wollebaeki Silverstsen, 1953). Kim y col (1975) mencionan la presencia de A. microchir para las especies de Otariidae del hemisferio norte, describiendo la misma especie para el hemisferio sur. Morejohn (1975) y Repenning (1975) proponen un origen monofilético en los grupos de Otariinae y Arctocephalinae, con lo cual los lobos marinos del Norte y los del sur serían descendencia uno del otro con una diferencia de unos dos millones de años.

Así, al poseer Eumetopias y Zalophus el parásito A. microchir y considerando la especificidad huésped-parásito (Atías 1998), al desarrollarse a partir de ellos el grupo hacia el hemisferio sur, podrían haber arrastrado al parásito hacia los géneros Otaria y Arctocephalus.

El lobo marino común del hemisferio sur (Otaria flavescens) posee una sola capa de pelo determinando períodos de buceo cortos, no sobrepasando los 20 minutos. Así el parásito chupador se ubicaría alrededor del hocico y las fosas nasales que son las primeras que emergen del agua. Secundariamente la densidad de la melena en los machos adultos permitiría incorporar aire en el sector anterior del cuerpo.

La creciente interacción del lobo marino con la población humana en diversas partes de Chile y el cono de América del Sur nos sugiere la necesidad de estudiar a estos insectos parásitos chupadores.

\section{RESUMEN}

Los ectoparásitos de los mamíferos marinos en Chile son poco conocidos. Se recolectaron individuos en animales adultos y jóvenes de una lobería reproductiva de lobo marino común (Otaria flavescens) en Isla Metalqui, de la Región de Los Lagos, Chile. Se identifican y describen los especímenes de Anoplura apoyado en claves existentes. Dicha descripción y la comparación con la evolución y distribución de la familia Otariidae en el mundo nos permite concluir que Antarctophthirus microchir es una especie descrita por primera vez en Chile y podría representar una posible evidencia de un origen monofilético de los grupos de Otariidae en los hemisferios norte y sur.

\section{REFERENCIAS}

Aguayo A, M Sepúlveda, H Díaz, F Palma, J Yáñez. 1998. Informe final proyecto FIP 96-51. Censo poblacional Lobo Marino común en el Litoral de la V a la IX Región de Chile. Fondo de Investigación Pesquera, Ministerio de Economía Chile.

Atías A. 1998. Parasitología Médica. Mediterráneo, Santiago, Chile.

Clay T, C Moreby. 1970. Mallophaga and Anoplura of subantarctic islands. Pacific Insects Monograph 23, 216-220.

Dailey M, R Ellin, A Parás. 2005. First report of parasites from pinnipeds in the Galapagos Islands, Ecuador, with a description of a new especies of Philophthalmus (Digenia: Philophthalmidae). J Parasitol 91, 614-617.

Fasio A, A Palmeira, M Méndez, S Battle, P Bornio. 2000. Interacción de lobos marinos de un pelo, Otaria flavescens, y la pesquería artesanal, Cabo San Antonio, Buenos Aires, Argentina. Anales de $9^{a}$ Reunión de trabajo de especialistas en Mamíferos Acuáticos de América del Sur, Buenos Aires, Argentina.

Gales N. 1995. Hooker's sea lion recovery plan. (Phocarctos hookeri). In: Threatened species recovery plan series $N^{o} 17$. Treatened Species Unit. Department of Conservation, Wellington, New Zealand.

Kim KC, CA Repenning, GV Morejohn. 1975. Specific antiquity of the sucking lice and evolution of Otariid seals. In: Biology of the seal Rapp P v Réun Cons Int Explor Mer 1, 69, 544-549.

Koen Alonso M, E Crespo, S Pedraza, N García, M Coscarella. 2000. Food habits of South American Sea Lion, Otaria flavescens, of Patagonia. Argentina. Fishery bulletin 98, 250-263.

Mehlhorn B, H Mehlhorn, J Plötz. 2002. Light and scanning electron microscopical study on Antarctophthirus ogmorhini lice from the Antarctic seal Leptonychotes weddellii. Parasitol Res 88, 651-660.

Morejohn GV. 1975. A phylogeny of Otariid seals based on morphology of the baculum. In: Biology of the seal. Rapp P v Réun Cons Int Explor Mer 1, 69, 49-56.

Murray MD, MSR Smith, Z Soucek. 1965. Studies on Ectoparasites seals and penguins. II. The ecology of the louse Antarctophthirus ogmorhini (Enderlein) on the Weddell seal, Leptonychotes weddelli, Lesson. Aust J Zool 13, 761-771.

Murray MD. 1976. Insect parasites of marine birds and mammals. In: Marine Insects, Chapter 4. Cheng L (ed.). North-Holland Publishing Company, The Netherlands, Pp 79-96.

Oliva D. 1988. Otaria byronia (de Blainville, 1820), the valid scientific name for the southern sea lion (Carnivora: Otariidae). J Nat Hist 22, 767-772.

Oliva D, W Sielfeld, R Durán, M Sepúlveda, MJ Pérez, L Rodríguez, W Stotz, V Araos. 2003. Interferencia de mamíferos marinos con actividad pesquera y de acuicultura. Informe final FIP 2003-32 del Fondo de Investigación Pesquera del Ministerio de Economía, Chile.

Repenning CA. 1975. Otarioid evolution. In: Biology of the seal. Rapp P v Réun Cons Int Explor Mer 1, 69, 27-33.

Sepúlveda M, D Oliva, F Palma. 2001. Daily and annual circarhythmus activity in the southern American Sea lion, Otaria flavescens (Carnivora, Otariidae) at the central Zone of Chile. Rev Biol Mar y Oceanogr 36, 181-187.

Szteren D, E Paez. 2002. Predation by Southern sea Lion (Otaria flavescens) and artesanal fishing catches in Uruguay. J Mar Freshw Res 53, 1161-1167. 